\title{
Comparison of Effectiveness of Two Designs of Interdental Toothbrushes in removing Dental Plaque
}

\author{
${ }^{1}$ Aruni Tilakaratne, ${ }^{2}$ Kathya L Perera, ${ }^{3}$ Vajira P Jayasinghe
}

\begin{abstract}
Aim: To compare the plaque removal ability of two designs of interdental toothbrushes (IDTBs) ["tube-like" IDTB = TT and "conical" IDTB $=\mathrm{CT}$ ] in the posterior part of the mouth in adult patients.
\end{abstract}

\begin{abstract}
Materials and methods: The study sample consisted of 107 adult patients who were in the periodontal maintenance phase having completed treatment for "chronic mild-moderate gingivitis." Each selected patient was subjected to plaque scoring in the posterior sextants. Four sites per tooth were scored after plaque disclosing. The percentage of plaque was calculated. One of the two designs of IDTBs (TT or CT) was randomly assigned for each patient. They were instructed to carry out eight strokes back-and-forth in the relevant interdental spaces, following demonstration of the technique on a model. The interdental surfaces were reexamined for remaining plaque, and the plaque score was taken. The percentage of reduction of plaque after using the IDTB was calculated.
\end{abstract}

Results: The mean interdental plaque score for the "TT design" group ( $n=56$ ) was $82 \%$ initially, whereas the same for "CT design" group ( $\mathrm{n}=51$ ) was $78 \%$, and this difference was not significant statistically. Following use of TT design, the plaque score in this group was reduced to $24 \%$, which is a $71 \%$ reduction from the initial plaque score. This reduction was significant statistically $(p<0.004$; t-test). The CT design also showed a dramatic reduction $(79 \%)$ in plaque score following use of this design ( $p<0.007$; t-test).

Conclusion: Although both designs (CT and TT) were highly effective in posterior interdental spaces, the CT design appeared to be better than TT design in controlling interdental plaque.

Keywords: Conical interdental toothbrush, Dental plaque, Gingivitis, Interdental space, Interdental toothbrush, Periodontitis, Tube-like interdental toothbrush.

${ }^{1}$ Professor, ${ }^{2}$ Trainee, ${ }^{3}$ Senior Lecturer

${ }^{1}$ Department of Oral Medicine and Periodontology, Faculty of Dental Sciences, University of Peradeniya, Peradeniya Sri Lanka

${ }^{2}$ Department of Periodontology, Faculty of Dental Sciences University of Peradeniya, Kandy, Sri Lanka

${ }^{3}$ Department of Prosthetic Dentistry, Faculty of Dental Sciences University of Peradeniya, Kandy, Sri Lanka

Corresponding Author: Aruni Tilakaratne, Professor Department of Oral Medicine and Periodontology, Faculty of Dental Sciences, University of Peradeniya, Peradeniya, Sri Lanka Phone: +0094812397450, e-mail: tlkrtn@yahoo.com
How to cite this article: Tilakaratne A, Perera KL, Jayasinghe VP. Comparison of Effectiveness of Two Designs of Interdental Toothbrushes in removing Dental Plaque. Int J Experiment Dent Sci 2017;6(1):17-21.

Source of support: This research was carried out with the generous sponsorship of George Steuart Company Ltd which provided the two designs of inter-dental toothbrushes for this study.

\section{Conflict of interest: None}

\section{INTRODUCTION}

Microbial plaque or dental plaque is well documented as the primary etiological factor in the development of chronic inflammatory gingivitis and periodontitis. The interproximal or interdental periodontal sites are more frequently affected with gingivitis and periodontitis. ${ }^{1}$ These sites are often coated with plaque for the reason that, conventional toothbrush (CTB) alone is relatively ineffective in reaching interproximal areas. However, the CTB is effective in removing plaque on buccal, oral, and occlusal surfaces. ${ }^{2}$ According to available literature, there has been an indication to believe that some $40 \%$ of dental surfaces are never reached with the CTB. ${ }^{3}$ Therefore, interproximal cleaning represents an important aspect of oral self-care in terms of maintaining proper gingival health, which, in turn, would help in the prevention of periodontal disease and caries. It has been widely held by the clinicians that most of their patients use a CTB as the only tool for daily plaque removal, and some patients are either unaware of a need to carryout interproximal cleaning or fail to follow advice regularly.

Interdental elements are frequently recommended by dental professionals in the developed world to their patients in order to achieve and maintain good standards in dental and periodontal health. Although there is no distinct or best method that would be apt for all the patients, a good interdental device requires penetration between the adjacent teeth. Floss, wood sticks, interdental brushes, rubber tip stimulators, and irrigating devices currently represent the primary methods of interproximal cleaning. ${ }^{1}$ Floss is the most widely recommended routine method of interdental cleaning, and the American Dental Association reports that up to $80 \%$ of interdental plaque can be removed by this method, resulting in a significantly reduced incidence of caries and periodontal disease. ${ }^{4}$ On the contrary, there is also evidence to believe that interdental brushing would be 


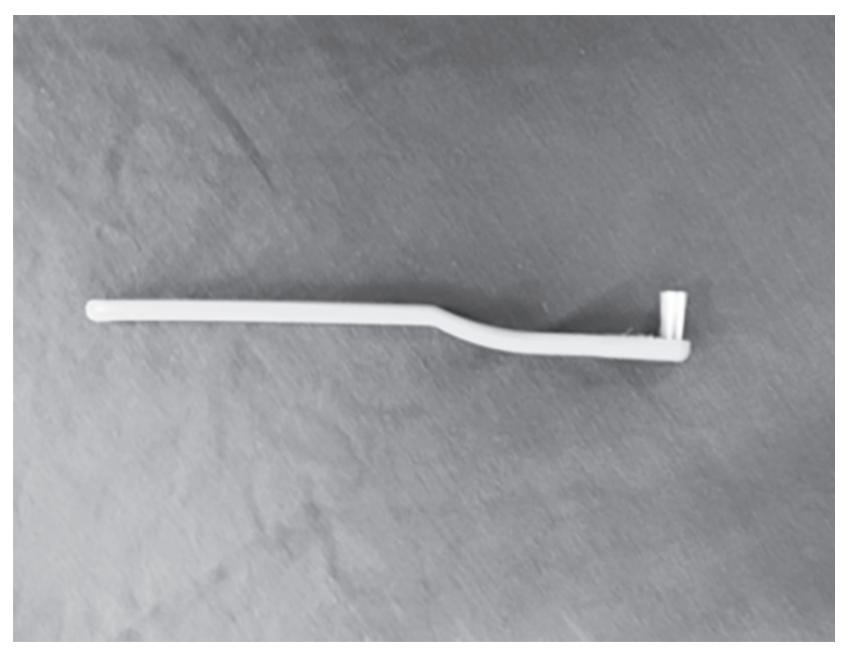

Fig. 1: Prepared IDTB

more effective than flossing in removing plaque as well as to obtain significant pocket depth reductions. ${ }^{2,5}$ However, it is not clear whether the reduction in pocket depth was directly due to any greater efficiency in plaque removal or due to mechanical depression of the interdental papilla with the use of the interdental brush, leading to marginal gingival recession. While dental floss can be effectively used to remove plaque in the anterior part of the mouth, its use in the interdental sites of the posterior teeth could be challenging due to difficult access and poor manipulative skills of the individual.

The clinical experience of dental professionals has proven true that most patients who are recommended to use dental floss would want to give it up since they find the technique of flossing as too demanding with regard to manual dexterity and time factor. Therefore, once recommended, the flossing technique would need much more monitoring, constant encouragement, and guidance given to patients compared with other interdental cleaning aids.

In view of the doubts regarding the user-friendliness of dental floss, interdental toothbrushes (IDTBs), which are specifically designed to be used in the interdental spaces of varying sizes (narrow to wide), can be made a suitable alternative for effective plaque control, particularly in the posterior part of the mouth. Since such specifically designed IDTBs are not widely available in the Sri Lankan market, most concerned clinicians recommend a "prepared IDTB" (which is called as a "prepared endtufted toothbrush"), made out of a small-headed CTB (Fig. 1). While this becomes fairly effective for some patients, there also are patients who seem not to improve their interdental plaque control ability (particularly in the posterior part of the mouth) with the prepared IDTB. However, recently in Sri Lanka, the specifically designed IDTBs have begun to emerge, particularly the "tubelike type" (TT) of the IDTB (Fig. 2). The next available

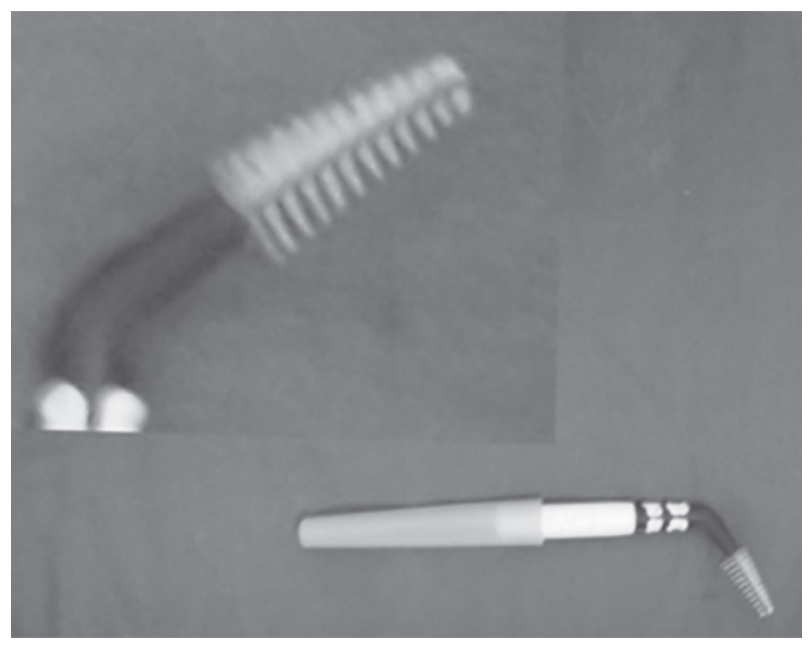

Fig. 2: Conical IDTB (CT design)

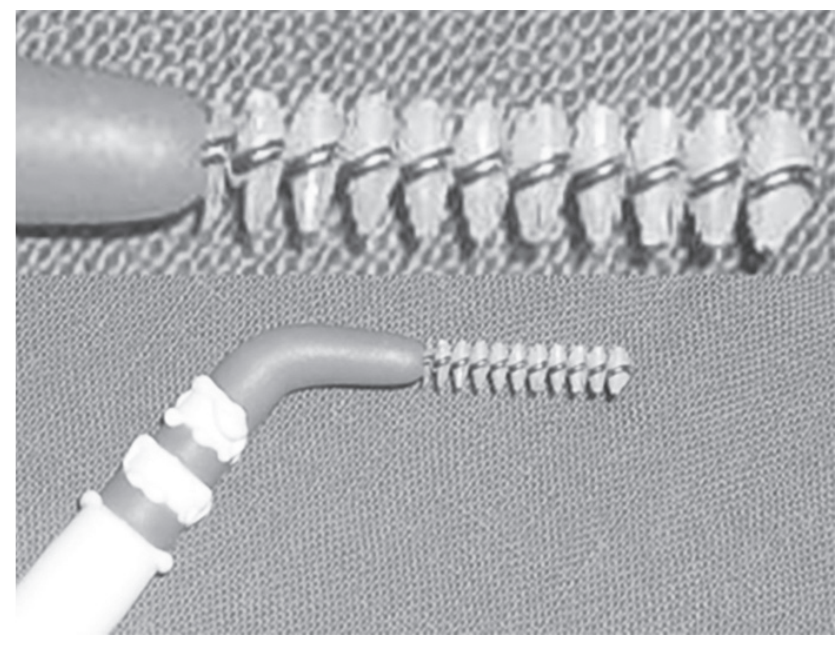

Fig. 3: Tube-like IDTB (TT design)

specifically designed IDTB, the "conical type" (CT) (Fig. 3) may also be effective in achieving a good plaque control if used at an angle in posterior interdental sites.

Clinicians in Sri Lanka reported that both the TT and CT could help in achieving better plaque removal compared with the prepared IDTBs. However, there is no clear understanding as to the best type of IDTB in varying situations in the posterior interdental spaces, more importantly, according to the size of the interdental space against the shape of the IDTB. Therefore, the aim of the present study was to compare the plaque removal ability of TT IDTB and CT IDTB in the posterior part of the mouth in a group of patients who have failed to acquire an acceptable plaque control by using prepared IDTBs up to the time of their periodontal maintenance phase (PMP).

\section{MATERIALS AND METHODS}

\section{Study Population}

The study was carried out in the Division of Periodontology, Faculty of Dental Sciences, University of Peradeniya, 
Sri Lanka, following approval of the Research Ethics Review Committee of the Faculty of Dental Sciences, University of Peradeniya. Patients, who turned up for the first appointment in their PMP, were randomly drawn from the PMP register maintained in the clinic. Each patient was then individually assessed to find out whether he/ she would fit into the criteria of the study given below. The maximum number of patients who fitted into the study with the inclusion and exclusion criteria was 107 , although the originally targeted sample size was 100 (according to the calculated patient numbers in the annual PMP patient register maintained in the clinic).

\section{Experimental Protocol}

The following were the inclusion and exclusion criteria and the basis for selection of the given patients and the tooth sites in this study.

\section{Inclusion Criteria}

- Patients should be between 20 and 40 years of age: (This age range was selected to minimize the variability with regard to manual dexterity).

- All patients should have been diagnosed as "chronic mild-moderate gingivitis" and should have undergone the "standard phase of active periodontal treatment" (SPAPT) within the previous 3 months.

- Each patient should have at least three (out of four) posterior sextants, which can be scored. There should be a minimum of four teeth in each posterior sextant for scoring, enabling assessment of plaque removal ability in at least three interdental spaces.

- All suitable posterior sextants for scoring should have revealed unsatisfactory plaque control (plaque score $>30 \%$ per sextant) at least on three occasions out of five treatment visits during SPAPT (judged by the clinical records).

- Any given sextant at the first PMP visit (plaquescoring visit), upon disclosing should reveal a minimum of $20 \%$ interdental plaque in order to qualify for scoring.

- All patients should have been right-handed.

\section{Exclusion Criteria}

- Any significant medical history which either complicates the periodontal disease presentation or affects the outcome of treatment

- Any known manual impairment

- Patients who have past experience in using IDTBs

- Any local plaque retentive factor which had not been corrected yet (e.g., malaligned teeth, crowding, teeth that are out of arch, anatomical defects, etc.)
Additionally, the following conditions were looked at, before selecting teeth or sextants for scoring. If the conditions were not met, either the sextant or the subject was excluded from the study.

- The condition of proximal tooth surfaces: These should have been caries-free. If they were with restorations, they should have mimicked physiological tooth surfaces with no overhangs, no underfillings or open margins. This was to avoid obstruction or altered access for the IDTBs due to ill-contouring or roughened proximal surfaces.

- The presence of very mobile or painful teeth. (This was to avoid any disturbance or obstruction when accessing the interdental spaces).

All subjects had already undergone SPAPT for the management of chronic mild-moderate gingivitis.

Standard phase of active periodontal treatment involved the following:

- First visit: Hygiene phase of periodontal therapy including home care methods involving

- Patient education, motivation, and oral hygiene instructions

- Disclosing plaque, recording plaque charts, and reinforcement of plaque control

- Plaque control methods - methods of basic oral hygiene, primarily with the standard, CTB, manual toothbrush. The systematic method of brushing was demonstrated on a model and then in the patient's mouth with his/her toothbrush.

- Special plaque control methods - prescribing a "prepared IDTB" (Fig. 1).

- Second visit: Plaque monitoring and mechanical debridement to remove plaque retentive factors supragingivally, which involved

- Plaque disclosing and recording plaque scores and reinforcing on plaque removal

- Full mouth ultrasonic scaling

- Third visit: Plaque monitoring and mechanical debridement to remove plaque retentive factors, which involved

- Plaque disclosing and recording plaque scores and reinforcing on plaque removal

- Subgingival scaling by using both ultrasonic and hand instruments

- Fourth visit: Plaque monitoring and mechanical debridement to remove plaque retentive factors, which involved

- Plaque disclosing and recording plaque scores and reinforcing on plaque removal

- Removal of other plaque traps, such as overhanging restorations, defective restorative work, open margins, inadequate contouring, or surface roughness on the interdental areas 
- Full mouth prophylaxis (removal of plaque, newly formed soft calculus and polishing).

- Fifth visit: Periodontal reevaluation, which involved

- Plaque disclosing and recording plaque scores and reinforcing on plaque removal

- Periodontal reevaluation

- Full mouth prophylaxis (if indicated)

The duration allowed between the first and second visits, second and third visits, and third and fourth visits was 1 week, whereas the duration between the fourth and fifth visit was 6 weeks; during which a periodontal reevaluation was carried out. Following this reevaluation (fifth) visit, the patients were recalled for the first periodontal maintenance visit in 2 weeks.

\section{Scoring of Interdental Plaque}

All patients who qualified to be included into the study were explained about the scope of the study at the first PMP appointment. Their informed consent was obtained prior to scoring and charting of plaque levels. On this visit, plaque was disclosed using erythrosine solution and the percentage calculated. [Plaque recording was done at four sites per tooth (mesiobuccal, mesiolingual, distobuccal, and distolingual)]. The design of the IDTB (TT or CT) was introduced to each patient on a random basis. Following a demonstration of the technique of interdental brushing on a model, each patient was guided to use the IDTB while looking at a mirror. The IDTB was first to be moved gently from a buccal-lingual direction twice, through the interdental space (if space was wide enough). Then it had to be similarly moved from lingual-buccal direction twice, through the interdental space in the same way. If the interdental space was narrow for the brush to go through, it had to be used along the gumline twice, accessing from buccal and lingual aspects in a similar manner.

The categorization of interdental spaces was as wide or narrow, based on the criteria as follows. Wide: The brush (bristled part) can be passed through the interdental space, only with slight resistance. Narrow: The bristled part of the brush cannot be passed through the interdental space and therefore, the interdental space was accessed for cleaning at an angle, without passing it through the interdental space.

Each patient thus carried out eight strokes back and forth in or around a given interdental space. All interdental surfaces in the sextant were examined for remaining plaque, and the plaque score was calculated. Thus, the percentage of reduction in the amount of plaque after using the interdental brush was calculated.

In order to overcome interexaminer variability, all examinations and measurements were performed by the same and well-trained examiner.

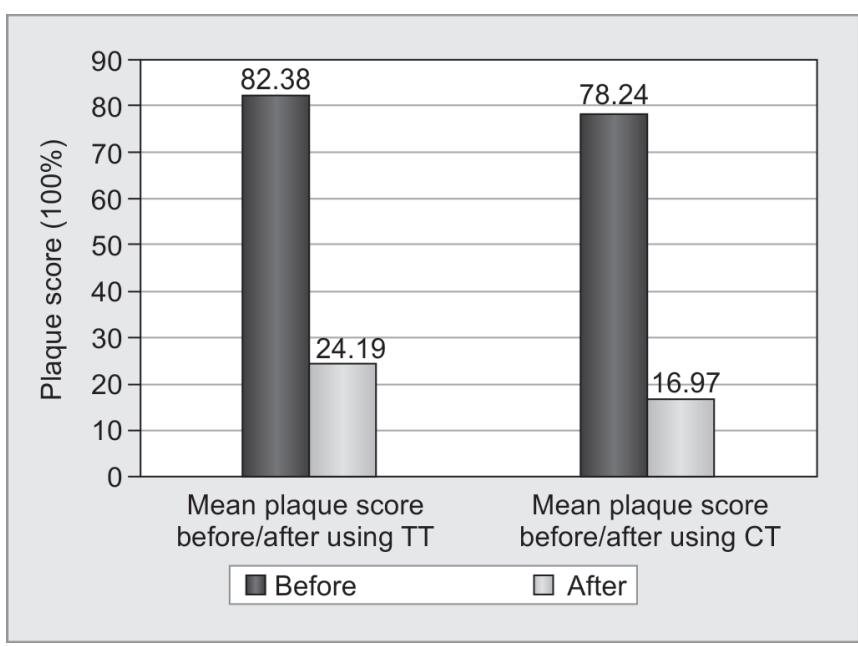

Graph 1: Mean interdental plaque scores for TT design group and CT design group before and after using toothbrushes

The collected data were statistically analyzed in order to achieve the objectives of the study.

\section{RESULTS}

The mean interdental plaque scores for the two groups (TT design group and CT design group) were compared before they were made to use the selected design of the IDTB (Graph 1). The mean plaque score for the TT design group ( $\mathrm{n}=56)$ was found as $82 \%$, whereas the same for CT design group $(n=51)$ was $78 \%$. This difference was not significant statistically when tested using t-test.

However, after using the TT design, the interdental plaque score in this group was reduced to $24 \%$ (from $82 \%$ ), which is a $71 \%$ reduction from the initial plaque score. This reduction was significant statistically $(\mathrm{p}<0.004$ as tested by t-test). Similarly, the group which was given CT design also showed a dramatic reduction (79\%) in plaque score after using the brush, and the score was reduced to $17 \%$ (from $78 \%$ ). This plaque reduction achieved by the CT design appeared to be significant statistically $(\mathrm{p}<0.007$, t-test). Thus, the interdental plaque reduction was $71 \%$ with the TT design, whereas the plaque reduction with the CT design was $79 \%$.

\section{DISCUSSION}

In a country like Sri Lanka, the use of dental floss as a home care plaque control method appears somewhat remote according to the routine lifestyles of most people. This is especially true with the rural and the suburban populations. There could be a variety of reasons for this, such as lack of awareness, lack of manual dexterity, inadequate information, lack of professionally delivered training and monitoring, cost factor, and lack of time and enthusiasm. Once recommended, the flossing technique needs to be monitored by the dental professional with 
regular dental appointments made with the patient. This involves a significant portion of time and commitment both by the patient and the clinician. Spending adequate chair-side time demanded by the teaching of flossing technique is unfortunately far from reality in a country like Sri Lanka, mainly due to the inadequate dentist: population ratio in the country. At the same time, flossing may pose difficulties when attempted on the posterior part of the mouth, and would be made less effective for most individuals. The present study revealed that the IDTBs can be made an effective tool to remove plaque from the interdental spaces without much difficulty for the patients. Moreover the chair-side time taken for teaching and monitoring the technique of interdental plaque removal with the IDTBs would be much less compared with the method of flossing. The findings of the present study revealed that the IDTBs are extremely effective in removing plaque from the interdental spaces in the posterior part of the mouth, both in narrow and wide interdental spaces. The findings of this study are further substantiated by the results of a randomized controlled trial by Jackson et al, ${ }^{5}$ which reports that there were significant improvements in baseline disease parameters in the "interdental brush group" compared with the "floss group" both at the end of the 6-week and 12-week reviews. The same trial ${ }^{5}$ also has demonstrated that the patients were able to improve clinical periodontal outcomes by interdental cleaning with IDTBs, even before carrying out root surface debridement. A systematic review supports the IDTB as an effective alternative to dental floss to control interproximal gingival inflammation and thus an important oral self-care device to be recommended for patients. ${ }^{6}$

From the findings of the present study, it is concluded that although both designs of IDTBs (TT and CT) were highly effective in interdental plaque removal in the posterior segments, the CT design appeared to be slightly better than the TT design in controlling plaque.

\section{ACKNOWLEDGEMENT}

Author would like to thank Dr Sanjeewa Kularatne for his valuable help in statistical analysis of data in this research

\section{REFERENCES}

1. Slot DE, Dorfer GE, Van der Weijden. The efficacy of interdental brushes on plaque and parameters of periodontal inflammation: a systematic review. Int J Dent Hyg 2008 Nov;6(4):253-264.

2. Christou V, Timmerman MF, Van der Velden U, Van der Weijden G. Comparison of different approaches of interdental oral hygiene: inter-dental brushes versus dental floss. J Periodontol 1998 Jul;69(7):759-764.

3. Schmage P, Platzer U, Nergiz I. Comparison between manual and mechanical methods of interproximal hygiene. Quintessence Int 1999 Aug;30(8):535-539.

4. Warren PR, Chater BV. An overview of established inter-dental cleaning methods. J Clin Dent 1996 Feb;7(3 Spec No):65-69.

5. Jackson MA, Kellett M, Worthington HV, Clerehugh V. Comparison of inter-dental cleaning methods: a randomized controlled trial. J Periodontol 2006 Aug;77(8):1421-1429.

6. Imai $\mathrm{PH}, \mathrm{Yu} \mathrm{X}$, MacDonald D. Comparison of inter-dental brush to dental floss for reduction of clinical parameters of periodontal disease: a systematic review. Can J. Dent Hyg 2012 Feb;46(1):63-78. 